\title{
Monomorphic ventricular tachycardia due to protease inhibitor intoxication by atazanavir
}

\author{
Byunghyun Kim', Kyung Su Kim² \\ 'Department of Emergency Medicine, Seoul National University Bundang Hospital, Seongnam, Korea \\ ${ }^{2}$ Department of Emergency Medicine, Seoul National University Hospital, Seoul, Korea
}

\begin{abstract}
Atazanavir is a protease inhibitor approved for use in combination with other antiretroviral drugs for the treatment of human immunodeficiency virus infection. Atazanavir and other protease inhibitors can sometimes induce corrected QT prolongation and ventricular arrhythmia. A 40-yearold man with no comorbidities, except human immunodeficiency virus 1 infection, presented with palpitations 3 days after an overdose of 150 caps of atazanavir, with suicidal intent. His initial electrocardiogram showed monomorphic ventricular tachycardia, and hyperbilirubinemia was observed in his initial blood test. Immediately after magnesium sulfate infusion, his ventricular tachycardia was converted into junctional bradycardia with prolonged corrected QT. After 3 days of close observation in the intensive care unit, the corrected QT prolongation and hyperbilirubinemia were normalized.
\end{abstract}

Keywords Atazanavir sulfate; Intoxication; Tachycardia, ventricular; Hyperbilirubinemia
elSSN: 2383-4625

Received: 1 November 2017

Revised: 9 January 2018

Accepted: 7 February 2018

Correspondence to: Kyung Su Kim Department of Emergency Medicine, Seoul National University Hospital, 101 Daehak-ro, Jongno-gu, Seoul 03080, Korea

E-mail: kanesu@gmail.com

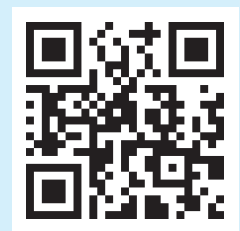

How to cite this article:

Kim B, Kim KS. Monomorphic ventricular tachycardia due to protease inhibitor intoxication by atazanavir. Clin Exp Emerg Med 2018;5(2):131-134.

This is an Open Access article distributed under the terms of the Creative Commons Attribution Non-Commercial License (http:// creativecommons.org/licenses/by-nc/4.0/). 


\section{INTRODUCTION}

In the era of highly active antiretroviral therapy (HAART), human immunodeficiency virus (HIV) infection is considered a chronic rather than a fatal disease.' HAART effectively prevents the development of acquired immune deficiency syndrome (AIDS) and opportunistic infections, thus providing HIV/AIDS patients an extended life expectancy with improved quality of life. ${ }^{2}$ Despite prolonged life expectancy, the suicide rate in HIV/AIDS patients is still higher than that in the general population. ${ }^{3}$ Especially in South Korea, misunderstanding and prejudice against HIV/AIDS patients leads to them suffering social isolation and stigmatization, thus causing depression and suicidal ideation. ${ }^{4}$ Patients with stable HIV infection are mainly managed in outpatient clinics with regular follow-up, and are prescribed long-term medications for more than a month. If the patients have any suicidal ideation, there could be a potential risk of poisoning through prescribed medications, especially using antiviral agents.

Atazanavir (Reyataz; Bristol-Myers Squibb, Princeton, NJ, USA), a protease inhibitor, is approved in many countries for use as a component of HAART regimens in the treatment of patients with HIV-1 infection. ${ }^{5}$ The most common adverse events of atazanavir are nausea, jaundice, and diarrhea. However, among less common adverse events, prolongation of the PR interval and corrected QT (OTc) have been reported in some patients. ${ }^{6}$ There have been 3 case reports of atazanavir-related torsades de pointes and 1 case report of ventricular tachycardia with QTc prolongation. ${ }^{7-9}$ The 4 patients involved had severe comorbidities, such as heart failure, liver cirrhosis, and intravenous drug usage. Here, we present the case of a 40-year-old HIV-1-infected man who presented with monomorphic ventricular tachycardia. He had no comorbidities, except for HIV-1 infection, and took 150 atazanavir capsules (200 $\mathrm{mg}$ atazanavir per capsule) with a suicidal intent.

\section{CASE REPORT}

A 40-year-old HIV-1-infected man presented to the emergency department with syncope and palpitations. He admitted to taking 150 atazanavir capsules (200 $\mathrm{mg}$ atazanavir per capsule) with the purpose of attempting suicide 3 days previously. His initial blood pressure was $119 / 75 \mathrm{mmHg}$, and his heart rate was 152 beats/min. The patient's initial 12-lead electrocardiogram (ECG) revealed ventricular tachycardia with prolonged QTc of $441 \mathrm{~ms}$ (Fig. 1A). Thirty minutes after a 10\% magnesium sulfate infusion
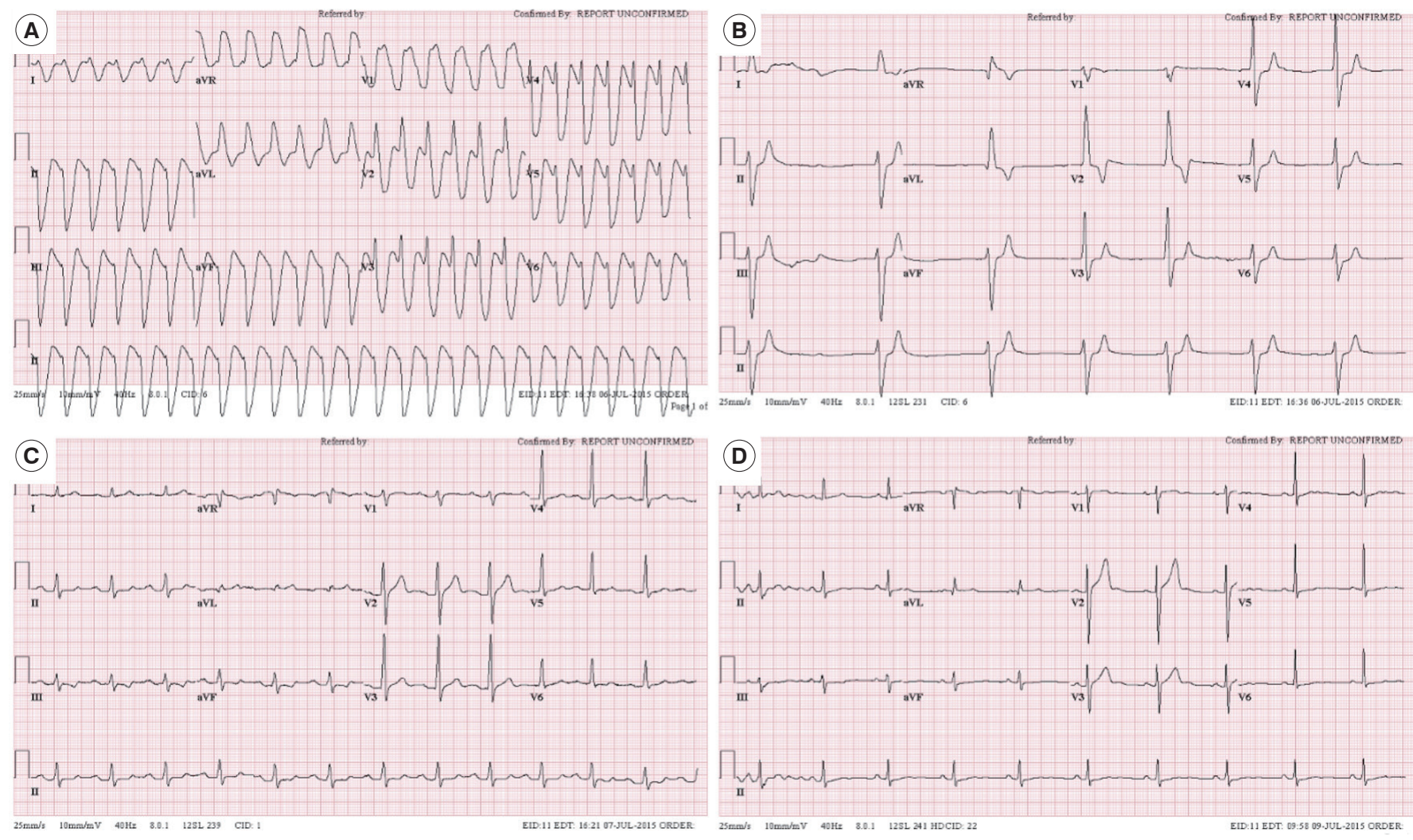

Fig. 1. Serial 12-lead electrocardiogram of patient with atazanavir intoxication. (A) Initial, (B) 30 minutes after $\mathrm{MgSO}_{4}$ infusion, (C) 6 hours after, and (D) on hospital day 3. 
Byunghyun Kim, et al.

Table 1. Laboratory and electrocardiogram data in a patient with atazanavir intoxication and monomorphic ventricular tachycardia

\begin{tabular}{lccccc}
\hline Parameter & Reference & Initial & After $12 \mathrm{hr}$ & After $24 \mathrm{hr}$ & After $48 \mathrm{hr}$ \\
\hline PR interval (msec) & $120-200$ & a) & 282 & 200 & 174 \\
Corrected QT time (msec) & $<430$ & 441 & 467 & 427 & 418 \\
Total bilirubin (mg/dL) & $0.2-1.2$ & 10.0 & 8.1 & 3.8 & 0.9 \\
\hline
\end{tabular}

${ }^{a}$ Not checked, due to ventricular tachycardia.

(2 g), his ECG rhythm converted to junctional bradycardia (Fig. 1B) with a heart rate of 41 beats/min, and the palpitations had improved. He was admitted to the emergency intensive care unit for close observation.

In his initial electrolyte panel, sodium, potassium, chloride, and total $\mathrm{CO}_{2}$ levels were $132,4.3,100$, and $16 \mathrm{mmol} / \mathrm{L}$, respectively. His initial serum magnesium level was $1.7 \mathrm{mEq} / \mathrm{L}$. Initial admission panel results were unremarkable, except for elevated total bilirubin $(10.0 \mathrm{mg} / \mathrm{dL})$ and creatinine $(1.94 \mathrm{mg} / \mathrm{dL})$. After 6 hours of observation and administration of normal saline $(80 \mathrm{~mL} / \mathrm{hr})$, his ECG rhythm converted to sinus rhythm with 1st degree atrioventricular block (Fig. 1C). During his hospital stay, his OTc time, PR interval and total bilirubin level decreased (Table 1). On day 2 of his hospital stay, a 2-D echocardiography undertaken by a cardiologist revealed that his ejection fraction was 65\%, but it did not reveal any other abnormalities. By day 3, his ECG had completely normalized (Fig. 1D) and the elevated total bilirubin level had decreased to $0.7 \mathrm{mg} / \mathrm{dL}$. He was discharged from the hospital general ward on day 3 , following a psychiatric consultation and an antiviral regimen change.

\section{DISCUSSION}

Prolongation of the QT interval for more than $500 \mathrm{~ms}$ increases the risk of torsades de pointes. ${ }^{10}$ Protease inhibitors, including atazanavir, and many other drugs cause QT prolongation through a blockade of the human ether-a-go-go-related gene potassium ion channel, which regulates a major repolarizing potassium current. ${ }^{11}$ Among the previous 4 cases with atazanavir toxicity in which torsades de pointes or ventricular tachycardia developed, one patient had congestive heart failure and was undergoing hemodialysis. ${ }^{7}$ Two patients used methadone, which also induces $\mathrm{OT}$ interval prolongation, and electrical defibrillation was delivered for the termination of torsades de pointes. ${ }^{8}$ In the last of the four cases, the patient had liver cirrhosis and doubled his usual dosage of oxycodone, which can induce a drug interaction with atazanavir, and electrical defibrillation was delivered for the termination of torsades de pointes. ${ }^{9}$

To our knowledge, this is the first case of atazanavir intoxication in an HIV patient with suicidal intent. Unlike the previous cases, in which electrical defibrillation was delivered for the termination of ventricular arrhythmia, we used $\mathrm{MgSO}_{4}$ as an initial antiarrhythmic drug, and soon after the $\mathrm{MgSO}_{4}$ infusion, the patient's ventricular tachycardia was terminated. In cases of torsades de pointes induced by drug intoxication, magnesium should be considered as the first antiarrhythmic drug. ${ }^{12}$ Magnesium can also be used in cases of sustained monomorphic ventricular tachycardia that are unresponsive to a first-line drug such as amiodarone. ${ }^{13}$ In the present case, we used magnesium as an initial antiarrhythmic drug because, in the brief period of initial assessment, we searched previous cases reports in which OTc prolongation was identified as a possible severe side effect of atazanavir use. Considering the patient's initial prolonged QTc interval, there was a risk of developing torsades de pointes or more severe ventricular arrhythmia. After the infusion of $\mathrm{MgSO}_{4}$, the patient's monomorphic ventricular tachycardia was converted to an atrioventricular nodal rhythm, and the patient's palpitation symptoms disappeared.

Uglietti et al. ${ }^{14}$ reported that the level of hyperbilirubinemia is related to the toxicity level of atazanavir in the early medication initiation period. We used the total bilirubin level as an indicator of serum atazanavir levels. As the patient's total bilirubin level decreased to a normal range, the PR interval and QTc time were also normalized.

In summary, monomorphic ventricular tachycardia was induced by atazanavir intoxication and was terminated soon after $\mathrm{MgSO}_{4}$ infusion. Through normal saline hydration and the withdrawal of atazanavir, the patient's QTc prolongation and hyperbilirubinemia were improved at the same time. In South Korea, where the number of HIV/AIDS patients is increasing and the suicide rate is high, physicians should be aware that poisoning using HAART medication can occur in susceptible patients, and QTc prolongation or ventricular arrhythmia can be induced by protease inhibitors.

\section{CONFLICT OF INTEREST}

No potential conflict of interest relevant to this article was reported. 


\section{REFERENCES}

1. Zlotorzynska M, Spaulding AC, Messina LC, et al. Retrospective cohort study of cancer incidence and mortality by HIV status in a Georgia, USA, prisoner cohort during the HAART era. BMJ Open 2016;6:e009778.

2. Samji H, Cescon A, Hogg RS, et al. Closing the gap: increases in life expectancy among treated HIV-positive individuals in the United States and Canada. PLoS One 2013;8:e81355.

3. Catalan J, Harding R, Sibley E, Clucas C, Croome N, Sherr L. HIV infection and mental health: suicidal behaviour: systematic review. Psychol Health Med 2011;16:588-611.

4. Kang $\mathrm{CR}$, Bang $\mathrm{JH}$, Cho $\mathrm{Sl}$, et al. Suicidal ideation and suicide attempts among human immunodeficiency virus-infected adults: differences in risk factors and their implications. AIDS Care 2016;28:306-13.

5. Croom KF, Dhillon S, Keam SJ. Atazanavir: a review of its use in the management of HIV-1 infection. Drugs 2009;69:110740.

6. Bristol-Myers Squibb. Reyataz product information [Internet]. Princeton, NJ: Bristol-Myers Squibb; 2017 [cited 2017 May 24]. Available from: https://packageinserts.bms.com/pi/pi_ reyataz.pdf.
7. Ly T, Ruiz ME. Prolonged OT interval and torsades de pointes associated with atazanavir therapy. Clin Infect Dis 2007;44: e67-8.

8. Gallagher DP, Kieran J, Sheehan G, Lambert J, Mahon N, MalIon PW. Ritonavir-boosted atazanavir, methadone, and ventricular tachycardia: 2 case reports. Clin Infect Dis 2008;47: e36-8.

9. Dabiesingh DS, Psevdos G, Sharp VL. Atazanavir-associated OT interval prolongation and torsades de points in a patient with liver cirrhosis: a rare but serious complication. Infect Dis Clin Pract 2011;19:226-8.

10. Priori SG, Schwartz PJ, Napolitano C, et al. Risk stratification in the long-OT syndrome. N Engl J Med 2003;348:1866-74.

11. Anson BD, Weaver JG, Ackerman MJ, et al. Blockade of HERG channels by HIV protease inhibitors. Lancet 2005;365:682-6.

12. Yap YG, Camm AJ. Drug induced OT prolongation and torsades de pointes. Heart 2003;89:1363-72.

13. Allen BJ, Brodsky MA, Capparelli EV, Luckett CR, Iseri LT. Magnesium sulfate therapy for sustained monomorphic ventricular tachycardia. Am J Cardiol 1989;64:1202-4.

14. Uglietti A, Novati S, Gulminetti R, Maserati R. Correlations between atazanavir $\mathrm{C}$ (trough) and hyperbilirubinemia: a case report. J Med Case Rep 2009;3:9307. 